\title{
Oxoglutaric aciduria
}

INSERM

\section{Source}

INSERM. (1999). Orphanet: an online rare disease and orphan drug data base.

Oxoglutaric aciduria. ORPHA:31

A rare, genetic, inborn error of metabolism disorder characterized by neonatal-onset of developmental delay, hypotonia, hepatomeg aly, lactic acidemia, increased creatine kinase levels, elevated alpha-ketog lutaric acid in urine, and a decreased plasma betahydroxybutyrate-to-acetoacetate ratio. Pyruvate dehydrogenase deficiency can be associated, leading to hypoglycemia and neurologic anomalies, including seizures. 